\title{
Long Physico-Chemical and Biological Monitoring for Treated Artificial Tidal Flats with Recycled Paper Sludge in Ago Bay, Japan
}

\section{Ahmed H.A. Dabwan ${ }^{1, a}$, Daizo Imai ${ }^{2}$, Satoshi Kaneco², Hideyuki Katsumata², Tohru Suzuki ${ }^{3}$, and Kiyohisa Ohta ${ }^{2}$}

\author{
${ }^{1}$ Faculty of Chemical Engineering Technology, Tati University College, Jalan Panchor, Teluk \\ Kalong, 24000 Kemaman, Terengganu, Malaysia
}

${ }^{2}$ Department of Chemistry for Materials, Graduate School of Engineering, Mie University, Tsu, Mie 514-8507, Japan

${ }^{3}$ Environmental Preservation Center, Mie University, Tsu, Mie 514-8507, Japan

aahmedmie2000@gmail.com (corresponding author)

Keywords: Recycled paper sludge ash, Long monitoring, physico-chemical, biological, artificial tidal flats, Ago Bay, Japan.

\begin{abstract}
Treated dredged sediment by using Hi Biah System (HBS) was used for the construction of five different artificial tidal flats (E1-E5) in Ago Bay, Japan. AGOCLEAN-P which is mainly made from paper sludge ashes was used as a coagulant and hardener. After the construction, continuous monitoring for the physico-chemical and biological parameters was carried out quarterly for 28 months from May 2005 to August 2007. Physico-chemical parameters measured were; particle size, loss on ignition (LOI), total organic carbon (TOC), chemical oxygen demand (COD), water content (WC), chlorophyll a, and acid volatile sulphide (AVS). At the end of the experiment, physico-chemical parameters for the artificial tidal flats along with the sandy natural flat (S2) were almost similar. In regards to the natural muddy flats (S1) the results showed remarkable increase in the particle size $<0.75 \mu \mathrm{m}$ throughout the experimental period, whereas insignificant decrease was observed for the medium particle size. In addition the area around station (S1) was characterized by high concentration of silt/clay around $75 \%$ for the particle size less than $75 \mu \mathrm{m}$. Biological parameter was represented by macrobenthos abundant as number of individuals, biomass, and species number. The abundant macrobenthos during the first year of monitoring was mollusca followed by polychaeta then bivalve, however at the end of the experiment, bivalve was the most dominant macrobenthos followed by mollusca and then polychaeta indicating that healthy environment was created.
\end{abstract}

\section{Introduction}

According to Mie Prefecture Department of Agriculture, Fisheries, Commerce and Industry, 2004, the annual pearl oyster production from Ago Bay is estimated to be 91 tons (dry weight), and a pearl oyster shell is estimated to be 821 tons. Although pearl oysters are not fed with formulated feeds, it is believed that the pearl farms are one of the important contributor to the organic enrichment of the under lying sediments of the bay by filtering phytoplankton and discharging feces in concentrated form [1]. In addition after the nucleus insertion into the oyster body, cleaning should be carried out weekly throughout all the cultivation period for nets and shells to remove accumulated dirt. Dredging the organically rich sediments is one of the ways to help ecosystem to achieve its prosperity. Usually water content in the dredged sediments is over $90-\mathrm{wt} \%$, thus transportation cost increases dramatically especially if the CDFs far away from the dredging activities. In order to reduce the water content from watery mud sediments Hi Biah System (HBS) was developed [2]. The water content of the dredged sediments was reduced from $90-\mathrm{wt} \%$ before treatment to $60-\mathrm{wt} \%$ after treatment by using AGOCLEAN-P as a coagulant.

The produced sediment from this innovated technology has been used in many applications such as; making granular micro-habitat beads for microorganism in order to treat contaminated seawater and polluted sediments. The sintered products made from dredged sediments fabricated at $400^{\circ} \mathrm{C}$ 
were found to be very effective adsorbents not only for the removal of heavy metals such as arsenic(III), cadmium(II), chromium(VI) and lead(II), but also good adsorbent for phosphate removal and hexavalnet chromium in aqueous solution $[3,4]$. Another application is creating stable surface for culturing seagrass such as Zostera marina. Sea bottom sediment was also used as a substitute for fine sand aggregate for the fabrication of concrete solids and marine reefs [5]. In constructing artificial tidal flats, the selection of a suitable sediment medium may be the primary factor in creating the sediment environment. For this reason mountainous sand alone for the construction of artificial tidal flats lacks a silt and clay components as well as organic matter, which is necessary to establish the same physic-chemical or biological structure as natural tidal flats [6]. In Ago Bay five artificial tidal flats were constructed mainly from treated dredged sediment mixed with natural sand from the same area and monitored for 20 months continuously. Results showed that the environmental conditions, number of benthos individuals and growth of short-necked clams in the artificial tidal flat were shown to be similar to those observed in a natural tidal flat [2].

This work is a continuation of the previous work where sampling was carried out for 20 months continuously plus seasonal data collected for the last 8 months for the physico-chemical properties and for the macrobenthos abundance. Data from other two nearby stations for natural mudflats (S1, S2) stations were characterized as muddy and sandy natural flats respectively were also monitored for the same parameters to get complete picture about the local macrobenthos community which represents important aquatic ecosystem health indicator.

\section{Experimental}

Study area, solidification method, constructed tidal flats building \& testing methods. Figure 1 shows the summary of tidal flats (E1-E5) construction method in Ago Bay. Ago Bay and constructed tidal flats (E1-E5) along with natural flats (S1 \& S2), hardeners used and other engineering details along with parameters analyzed and Ago Bay location have been described somewhere else [2].

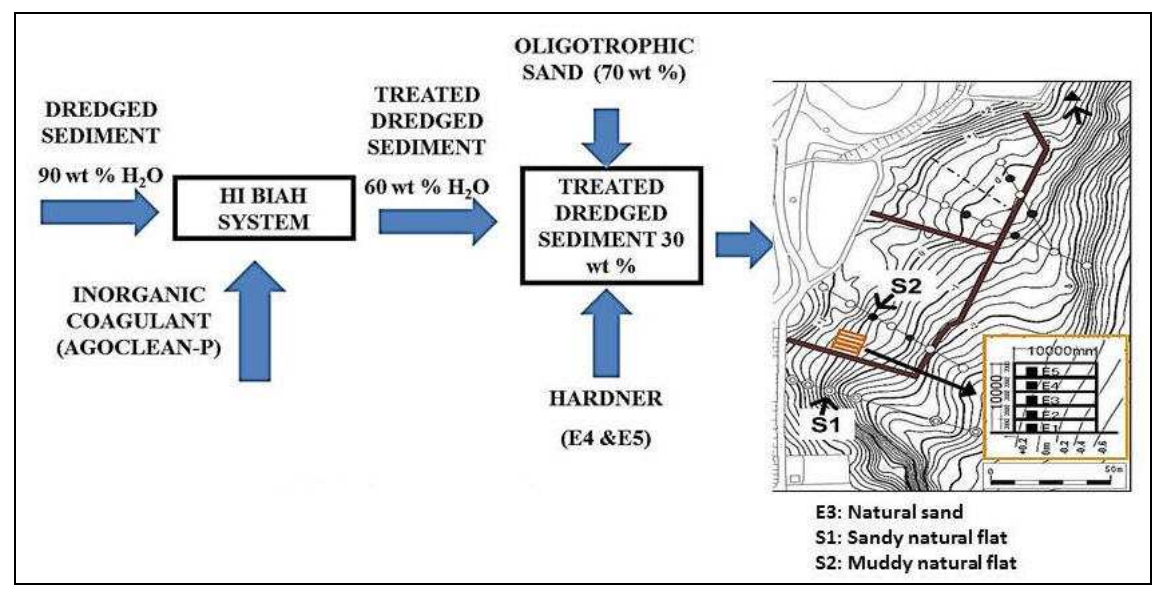

Fig. 1 : Tidal flats construction flow chat

\section{Results and Discussion}

Phsyco-chemical long monitoring parameters. Monitoring of the physico-chemical environmental conditions on the constructed tidal flats was carried out seasonally (every 3 months) for 28 months from May 28, 2005 to August 22, 2007. A full detail of the first 20 months of continuous monitoring was published earlier [4].

Starting with particle size analysis, the time series monitoring for the constructed artificial tidal flats (E1-E5) showed a dissimilarity for the particle size $<0.75 \mu \mathrm{m}$ and for the medium particle size for the first 9 months of monitoring (till Feb 2006) as shown in Fig. 2a. Natural flat (S2) shows the same pattern. After this period the graph became somehow stable. Range of the size particle from all artificial flats (E1-E5 along with S2) was between 15 and $45 \%$ with particle size less than $75 \mu \mathrm{m}$. 
At the end of the experiment there was no significant variation in the median particle size as shown in Fig. 2b. It is interesting to notice that higher values were observed for flat E5 for the first 3 months and E2 for the first 6 months (Fig.2a \& b) due to the flats preparation methods where steel sludge was used as hardening materials with ball shape in E5, at the same time pellets shape $(\sim 3 \mathrm{~cm}$ $\mathrm{L} \& 1 \mathrm{~cm} \mathrm{~d}$ ), were prepared in E2 with inorganic coagulant (AGOCLEAN-P) made mainly made from paper sludge ashes.

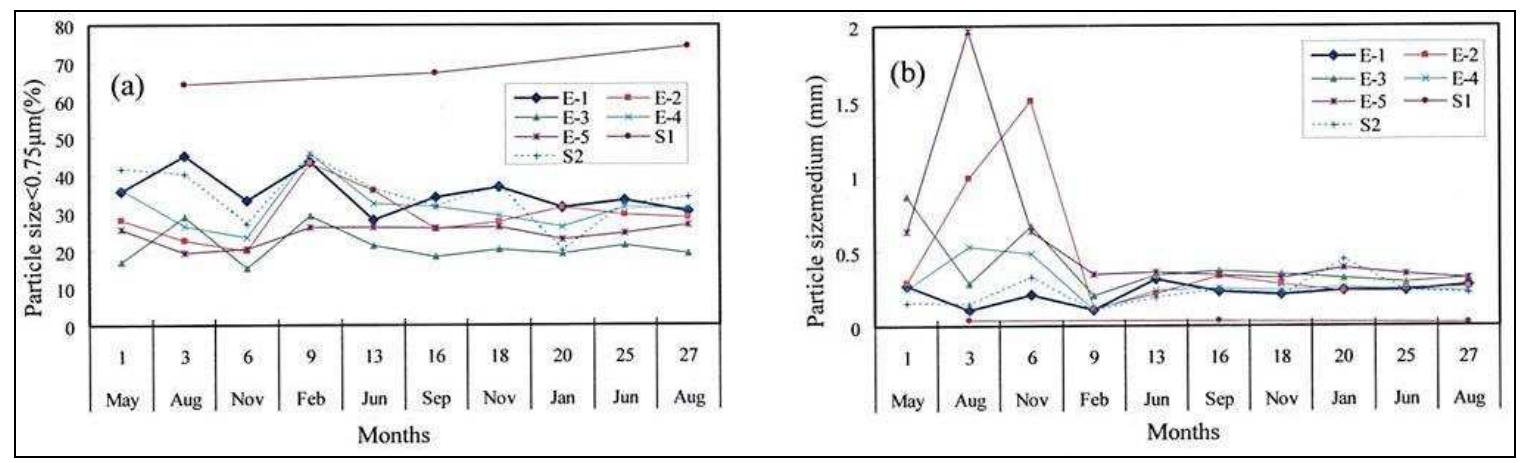

Fig. 2 : Particle size analysis of the artificial (E1-E5) and natural tidal flats (S1-S2).

The seasonal variation on phsyco-chemical parameters such as; loss on ignition (LOI), total organic carbon (TOC), and chemical oxygen demand (COD) give a very good indication about the organic matters in the sediments therefore almost same pattern was obtained for the three mentioned parameters for the artificial flats (E1-E5) plus natural station (S2) as shown in Figure 2. The minimum LOI was observed for the flat made from oligotrophic sand (no treatment with HBS), TOC \& COD show the same pattern for the same study period. Water content (WC) varied between $18 \sim 50 \%$ for the artificial flats whereas for the natural was in general higher. Benthic growth strongly connected with the existence of chlorophyll a [6]. After 18 months of construction, chlorophyll a reached its maximum values for all flats after that slight reduction was noticed (Fig.3). The amount of acid volatile sulphide (AVS) in sediments serves as a critical parameter in determining metal bioavailability and toxicity [7], that was high for muddy natural flat (S1) at zero time. After 28 months of continuous monitoring, AVS was kept within reasonable values (between 0.28 and $0.09 \mathrm{mg} / \mathrm{g}$ for the artificial flats and natural sandy flat S2), whereas for S1 the AVS value was $0.55 \mathrm{mg} / \mathrm{g}$.

Macrobenthos long monitoring Sampling was conducted in quarterly basis from May 2005 till August 2007 (Fig. 4 a-c). Three months after construction the population of macrobenthos in the artificial tidal flats (E1-E5) showed gradual increase throughout the experimental period compared to the natural ones $(\mathrm{S} 1, \mathrm{~S} 2)$ except in few occasions where the total number of macrobenthos was similar or lower (Fig. 4a). Even though low wet biomass of macrobentos was observed at all artificial and natural flats during June \& September 2006 (Fig. 4b), the abundance of macrobenthos as individuals $/ 0.1 \mathrm{~m}^{2}$ and number of species were not affected indicating that large macrobenthos such as bivalve were minority and the abundance macobenthos was mullusca (Fig. 4 a-c). It is worthy to note that there was high correlation between the obtained high values for the AVS and TOC during September 2006 (Fig. 3) and the sudden decrease in the wet biomass (Fig. 4c) indicating that the high concentrations of TOC and AVS might be the primary factors affecting the types of macrobenthos.

The dominant species in the first year were mollusca followed by polychaeta then bivalve. However at the end of the monitoring period it was notable that bivalve was the dominant species, coming next Mollusca, followed by polychaeta. The reason for this phenomenon could be attributed to the food net in the marine ecosystem where microalgae are the primary producer in the tidal flat, at the same time arthropoda, mullusca and polychaeta probably the main food providers for large crustaceans. 


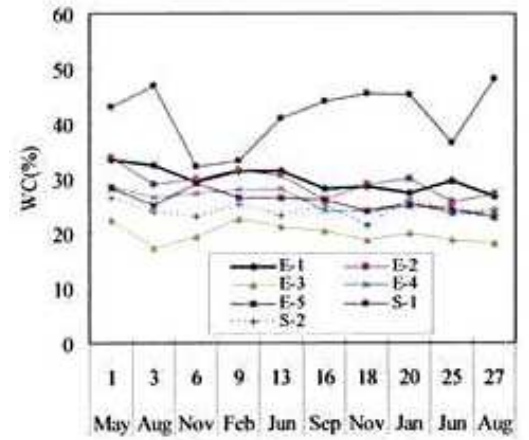

(a)

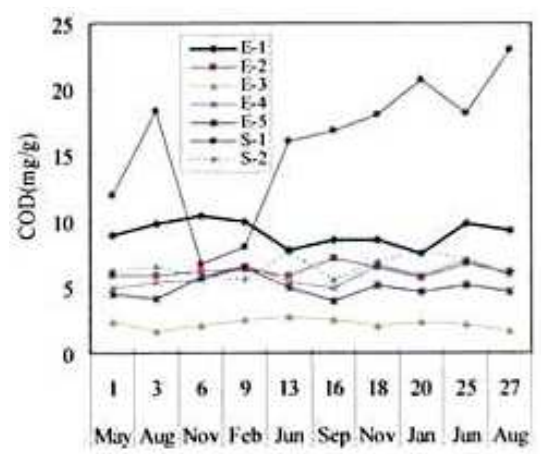

(d)

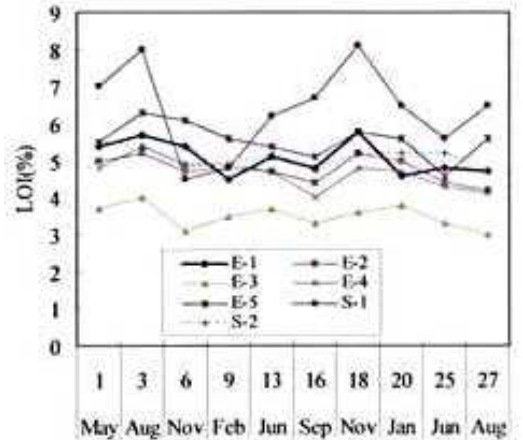

(b)

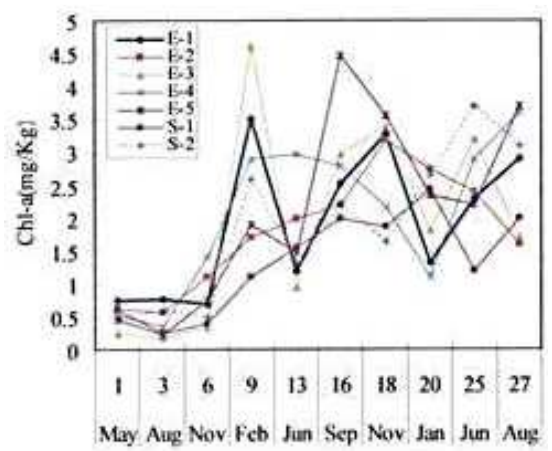

(e)

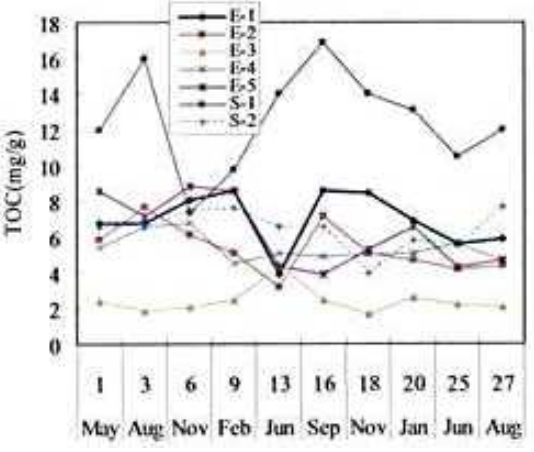

(c)

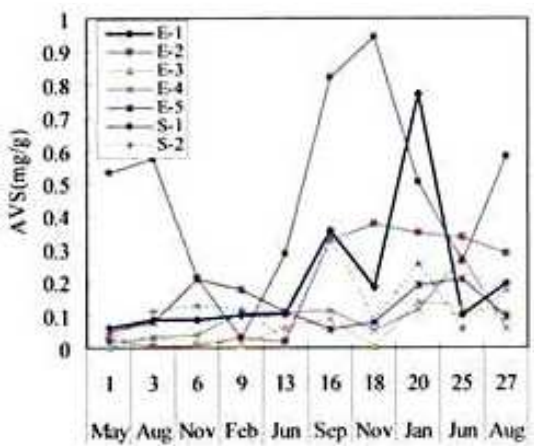

(f)

Fig. 3 : Seasonal changes in the chemical parameters for the artificial (E1-E5) and natural tidal flats (S1-S2) (a) Water content (WC); (b) loss on ignition (LOI); (c) Total organic carbon (TOC); (d) chemical oxygen demand (COD); (e) chlorophyll a; and (f) acid volatile sulphide (AVS).

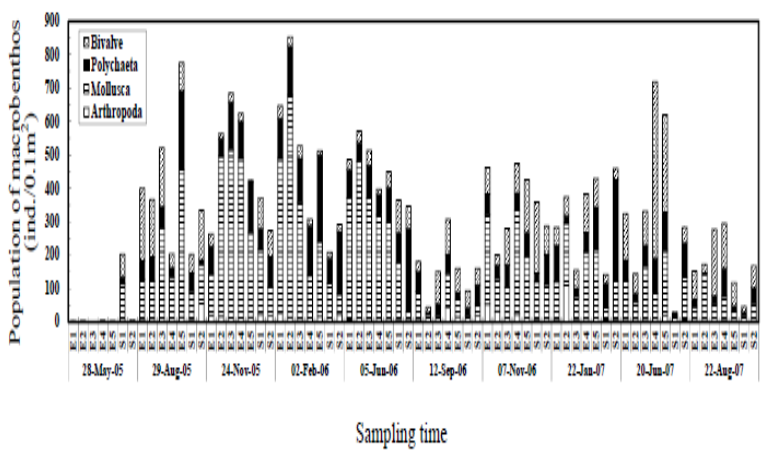

( a )

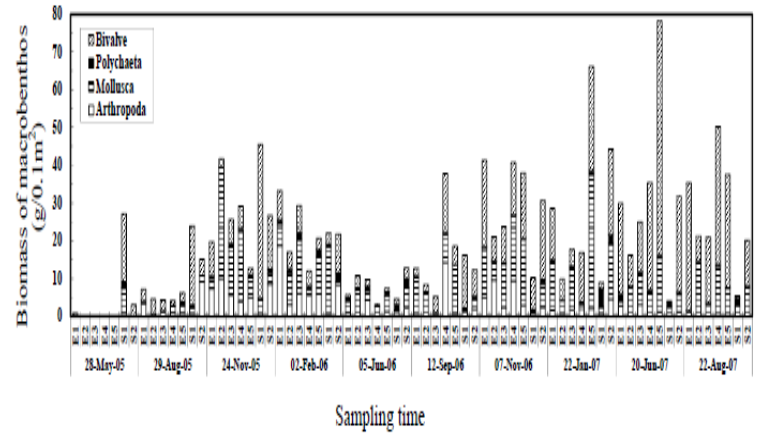

( b )

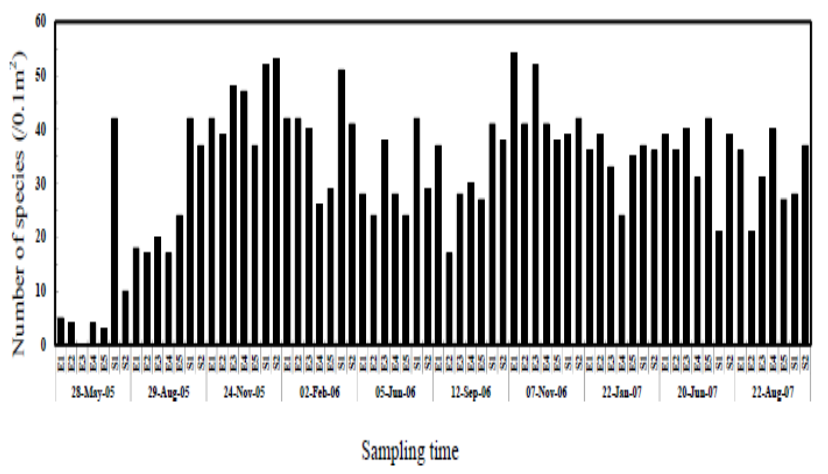

( c )

Fig. 4 : Seasonal variations of the macrobenthos for the artificial (E1-E5) and natural tidal flats (S1$\mathrm{S} 2$ ); (a) in the abundance (number of individuals); (b) in the biomass; (c) in species composition 


\section{Conclusion}

Conclusion derived from this long monitoring work can be summarized as follows; particle size at the end of the experiment for the tides treated by AGOCLEAN-P reached almost the same level for the artificial flats along with sandy natural flat, whereas muddy natural flat was higher in silt/clay and lower in the average particle size. The artificial tidal flats had lower content of LOI, TOC, and AVS. In contrast, chlorophyll a was higher for the artificial flats throughout the monitoring period. Macrobenthos population after 3 months increased gradually for the artificial flats having higher individual numbers compared to natural. Different pattern was noticed for wet weight biomass and number of species. In the first year both values were higher in the natural mudflats, after that reverse results were noticed. The outcome results from this long monitoring towards enhancement the biological productivity was visible based on the environmental parameters tested. Furthermore, toxicity of the coagulant and/or hardeners used in the tidal flats construction was negligible.

\section{Acknowledgments}

This work was performed as part of a joint collaboration research project entitled, "Environmental Restoration Project on the Enclosed Coastal Seas, Ago Bay", supported by CREATE (Collaboration of Regional Entities for the Advancement of Technological Excellence) organized by the Japan Science and Technology (JST) Agency. The main research was partly supported by the Ministry of Education, Culture, Sports, Science, and Technology of Japan. Parts of the experiments were conducted at Mie University, Japan and Tati University College, Malaysia. Any opinions, findings, conclusions, or recommendations expressed in this paper are those of the authors and do not necessarily reflect the view of the supporting organizations.

\section{References}

[1] A.B. Patel, Benthic denitrification and organic matter mineralization in intertidal flats of an enclosed coastal inlet, Ago Bay, Japan, Marine Poll. Bull. 57(2008) 116-124.

[2] D. Imai, S. Kaneco, H. Katsumata, K. Ohta, A.H.A Dabwan, T. Suzuki, T. Kato, Construction of biologically productive artificial tidal flats with solidified sea bottom sediments. Int. J. Soil Sediment Water. (2008) 2/1.

[3] K. Nakahama, S. Kaneco, H. Katsumata, T. Suzuki, A.H.A. Dabwan, K. Ohta, Removal of chromium (VI) from aqueous solution bysSintered material with sea bottom sediment and titanium dioxide, Photo/Electrochem. \& photobiol. Environ. energy \& fuel, (2007).

[4] K. Nakahama, M. Nakano, D. Imai, A. H. A. Dabwan, S. Kaneco, T. Suzuki, H. Katsumata, K. Ohta, Bacteria immobilized on porous sintered materials from sea bottom sediments: Fundamental Study. ITE Lett. Batteries New Technol. Med. 8/6, (2007) 8/6.

[5] D. Imai, A.H.A. Dabwan, S. Kaneco, H. Katsumata, T. Suzuki, M.Z. Hossain, K. Nakashima, T. Kato, K. Ohta, Use of sea bottom sediments for the fabrication of concrete solids. CIGR Ejournal. (2008), vol.10.

[6] R. Ishii, Y. Nakano, S. Nakai, W. Nishijima, M. Okada, Benthic ecosystem development in an artificial tidal flat constructed from dredged spoil. Marine Pollution Bulletin, 56 (2008) 20592066.

[7] O. Campana, A. Rodríguez, and J. Blasco, Identification of a potential toxic hot spot Associated with AVS spatial and seasonal variation, Arch. Environ. Contam. Toxicol. 56 (2009) 416-425. 